\title{
Evolution of deformation in neutron-rich Ba isotopes up to $A=150$
}

R. Lică,${ }^{1,2}$ G. Benzoni, ${ }^{3,{ }^{*}}$ T. R. Rodríguez, ${ }^{4}$ M. J. G. Borge,${ }^{1,5}$ L. M. Fraile, ${ }^{6}$ H. Mach,,${ }^{7, \dagger}$ A. I. Morales,${ }^{3,8,9}$ M. Madurga, ${ }^{1}$ C. O. Sotty, ${ }^{10,+}$ V. Vedia, ${ }^{6}$ H. De Witte, ${ }^{10}$ J. Benito, ${ }^{6}$ R. N. Bernard, ${ }^{4,11}$ T. Berry, ${ }^{12}$ A. Bracco,,${ }^{3,8}$ F. Camera,,${ }^{3,8}$ S. Ceruti,,${ }^{3,8, \S}$ V. Charviakova, ${ }^{7}$ N. Cieplicka-Oryńczak, ${ }^{3,13}$ C. Costache, ${ }^{2}$ F. C. L. Crespi, ${ }^{3,8}$ J. Creswell, ${ }^{14}$ G. Fernandez-Martínez, ${ }^{15}$ H. Fynbo, ${ }^{16}$ P. T. Greenlees, ${ }^{17,18}$ I. Homm, ${ }^{15}$ M. Huyse, ${ }^{10}$ J. Jolie, ${ }^{19}$ V. Karayonchev, ${ }^{19}$ U. Köster, ${ }^{20}$ J. Konki, ${ }^{17,18}$ T. Kröll, ${ }^{15}$ J. Kurcewicz, ${ }^{1}$ T. Kurtukian-Nieto, ${ }^{21}$ I. Lazarus, ${ }^{14}$ M. V. Lund ${ }^{16}$ N. Mărginean, ${ }^{2}$ R. Mărginean, ${ }^{2}$ C. Mihai, ${ }^{2}$ R. E. Mihai, ${ }^{2}$ A. Negret, ${ }^{2}$ A. Orduz,${ }^{22}$ Z. Patyk,${ }^{7}$ S. Pascu, ${ }^{2}$ V. Pucknell,,${ }^{14}$ P. Rahkila, ${ }^{17}, 18$ E. Rapisarda, ${ }^{1}$ J. M. Regis, ${ }^{19}$ L. M. Robledo, ${ }^{4}$ F. Rotaru, ${ }^{2}$ N. Saed-Samii, ${ }^{19}$ V. Sánchez-Tembleque, ${ }^{6}$ M. Stanoiu, ${ }^{2}$ O. Tengblad,${ }^{5}$ M. Thuerauf,,${ }^{15}$ A. Turturica, ${ }^{2}$

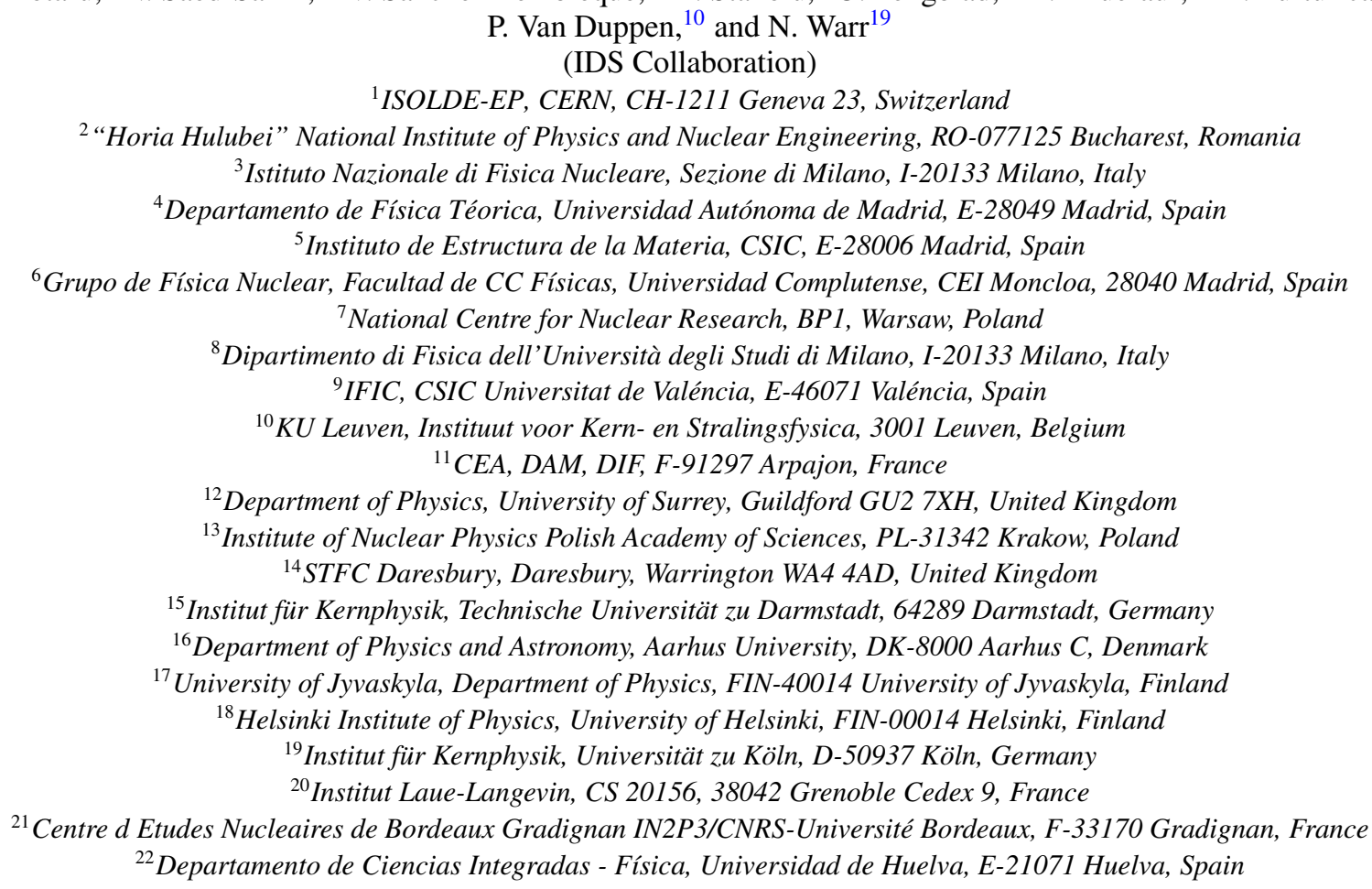

(Received 3 July 2017; published 5 February 2018)

\begin{abstract}
The occurrence of octupolar shapes in the Ba isotopic chain was recently established experimentally up to $N=90$. To further extend the systematics, the evolution of shapes in the most neutron-rich members of the $Z=56$ isotopic chain accessible at present, ${ }^{148,150} \mathrm{Ba}$, has been studied via $\beta$ decay at the ISOLDE Decay Station. This paper reports on the first measurement of the positive- and negative-parity low-spin excited states of ${ }^{150} \mathrm{Ba}$ and presents an extension of the $\beta$-decay scheme of ${ }^{148} \mathrm{Cs}$. Employing the fast timing technique, half-lives for the $2_{1}^{+}$level in both nuclei have been determined, resulting in $T_{1 / 2}=1.51(1) \mathrm{ns}$ for ${ }^{148} \mathrm{Ba}$ and $T_{1 / 2}=3.4(2) \mathrm{ns}$ for ${ }^{150} \mathrm{Ba}$. The systematics of low-spin states, together with the experimental determination of the $B\left(E 2: 2^{+} \rightarrow 0^{+}\right)$ transition probabilities, indicate an increasing collectivity in ${ }^{148-150} \mathrm{Ba}$, towards prolate deformed shapes. The experimental data are compared to symmetry conserving configuration mixing (SCCM) calculations, confirming an evolution of increasingly quadrupole deformed shapes with a definite octupolar character.
\end{abstract}

DOI: 10.1103/PhysRevC.97.024305

*giovanna.benzoni@mi.infn.it

†Deceased.

†Current address: "Horia Hulubei” National Institute of Physics and Nuclear Engineering, RO-077125 Bucharest, Romania.

${ }^{\S}$ Current address: KU Leuven, Instituut voor Kern- en Stralingsfysica, 3001 Leuven, Belgium.
Published by the American Physical Society under the terms of the Creative Commons Attribution 4.0 International license. Further distribution of this work must maintain attribution to the author(s) and the published article's title, journal citation, and DOI. 


\section{INTRODUCTION}

Nuclear interactions within the atomic nucleus can be understood in terms of a monopole part that contains the spherical mean field (spherical single-particle levels) and a multipole part that includes everything else [1]. The main contributions to the latter term come from the lowest order multipoles (quadrupole and octupole). Hence, the competition between the spherical mean field and these multipole contributions in a particular nucleus produces its intrinsic nuclear shape (spherical, quadrupole and/or octupole deformed, etc.). Barium isotopes $(Z=56)$ are located in a region of the Segrè chart characterized by a variety of shape-related phenomena, including shape coexistence and possible static octupole deformation. High order deformations can have a strong influence on $\gamma$-decay rates and on quasiparticle energies, which are, in turn, inputs for the various theoretical models developed to describe nuclei in this region [2,3].

The evolution of the nuclear shape with the number of nucleons is usually studied from mean-field calculations of potential energy surfaces (PESs) defined along multidimensional deformation spaces. In the Ba isotopic chain $(Z=56)$ microscopic-macroscopic [4] and fully microscopic HartreeFock-plus-BCS and/or Hartree-Fock-Bogoliubov (HFB) [5-7] calculations have been carried out, exploring both quadrupole and octupole degrees of freedom. It is noted that stronger octupole correlations are seen in the lightest $A=112-114$ and heaviest elements of the chain, starting from $A=144$, which corresponds to the well-known region of octupole deformation $N=56$ and $N=88$ [2]. Low-lying negative-parity states have also been found in ${ }^{122-126} \mathrm{Ba}$, whose character is understood in terms of octupole correlations [8].

The $\mathrm{Ba}$ isotopic chain has been extensively studied in past years up to $A=148$, mainly via spontaneous fission of ${ }^{252} \mathrm{Cf}$ and ${ }^{248} \mathrm{Cm}$ sources [9,10]. Spins and parities of both yrast and side bands could be assigned in most cases through directional correlations from oriented states (DCO) and polarization measurements. Population of excited nonyrast states in $\mathrm{Ba}$ isotopes via the $\beta$ decay of the $\mathrm{Cs}$ parent nuclei was also studied prior to the present experiment [11].

Most recently, ${ }^{144} \mathrm{Ba}$ and ${ }^{146} \mathrm{Ba}$ isotopes were studied at the CARIBU facility in Argonne. In both cases, the beam was post-accelerated to allow for a safe Coulomb excitation experiment, leading, for the first time, to the measurement of the $B\left(E 3 ; 3^{+} \rightarrow 0^{+}\right)$transition probabilities $[12,13]$. The $\beta$ decay of ${ }^{146} \mathrm{Cs}$ into ${ }^{146} \mathrm{Ba}$ was also measured with improved statistics, leading to a great extension of the level scheme up to $2.2 \mathrm{MeV}$ [14].

Excitation energies and electromagnetic properties (transition probabilities and moments) cannot be obtained within a pure mean-field description. Therefore, a direct comparison with the experimental data requires further developments. Recently, beyond-mean-field methods including symmetry restorations (particle number, angular momentum, and parity) and configuration mixing within the generator coordinate method (GCM) were implemented with relativistic [15] and Gogny interactions [16]. This symmetry conserving configuration mixing (SCCM) method included for the first time the interplay between axial quadrupole and octupole degrees of freedom on an equal footing. One of its first applications was the study of the spectra of ${ }^{144-146} \mathrm{Ba}$, providing a good agreement with the experimental data.

This paper presents the first experimental study of the excited structure of ${ }^{150} \mathrm{Ba}$ and an extension of the level scheme of ${ }^{148} \mathrm{Ba}$, together with the determination of transition probabilities in both nuclei. A theoretical interpretation will also be given in terms of quadrupole-octupole coupling. The paper is organized as follows: experimental details are given in Sec. II, while in Sec. III the proposed experimental level schemes for both ${ }^{148} \mathrm{Ba}$ and ${ }^{150} \mathrm{Ba}$ are shown. The comparison with SCCM theoretical results is given in Sec. IV and finally conclusions are drawn in Sec. V.

\section{EXPERIMENT}

In this work we study the ${ }^{148,150} \mathrm{Ba}$ isotopes, populated in the $\beta$ decay of their parent ${ }^{148,150} \mathrm{Cs}$ isobars. Cs isotopes were produced at ISOLDE (CERN) $[17,18]$ by fission of a nanostructured $\mathrm{UC}_{x}$ target induced by the $1.4 \mathrm{GeV}$ proton beam delivered by the PS Booster (PSB). The Cs atoms, thermally diffused out of the target matrix, were surface ionized and separated using the ISOLDE General Purpose Separator (GPS). The proton beam current during the run was 1.5-2 $\mu \mathrm{A}$. Intensities of the exotic beams reaching the experimental setup ranged from $5.5 \times 10^{2}$ ions $/ \mu \mathrm{C}$ for mass $A=148$ to 2 ions $/ \mu \mathrm{C}$ for the $A=150$ beam (beamline transmission of $\sim 70 \%$ ).

Cs ions were implanted on an aluminized mylar tape at the center of the detection setup. The beam extraction was started at the arrival of the proton pulse. The data acquisition recorded the arrival time of each proton pulse, using it as a reference for building decay curves. The tape was moved every $20-25$ proton pulses (corresponding to a PSB supercycle of $\sim 1$ minute) in order to remove the unwanted daughter activity.

The experimental setup consisted of the Isolde Decay Station (IDS), equipped with three fast-responding plastic scintillator detectors, to detect the $\beta$ particles, four high-purity germanium (HPGe) clover detectors for the detection of $\gamma$ rays following internal decay in the daughter nuclei, and three small-volume conic $\mathrm{LaBr}_{3}(\mathrm{Ce})$ detectors to perform lifetime measurements of specific nuclear states. The $\beta$ detection array efficiency was $20 \%$ while the total HPGe efficiency, after add-back, amounted to $6 \%$ at $0.6 \mathrm{MeV}$. The digital processing of the energy signals provides resolutions at $1.3 \mathrm{MeV}$ of the order of $2.3 \mathrm{keV}$ for the HPGe detectors and $40 \mathrm{keV}$ for the $\mathrm{LaBr}_{3}(\mathrm{Ce})$ ones. To increase the efficiency of the $\gamma$ array, no anti-Compton shield was used, with HPGe crystals placed at few centimetres from the implantation point. The collected spectra show peaks due to partial depositions of energy by $\gamma$ rays interacting via Compton scattering or pair-production mechanisms.

The setup was optimized to perform the evaluation of the lifetime of specific nuclear states using the fast-timing technique, which is based on the measurement of the time difference between a transition feeding and a transition deexciting a specific nuclear level. The transitions are detected in two different detectors, and the time difference is measured 


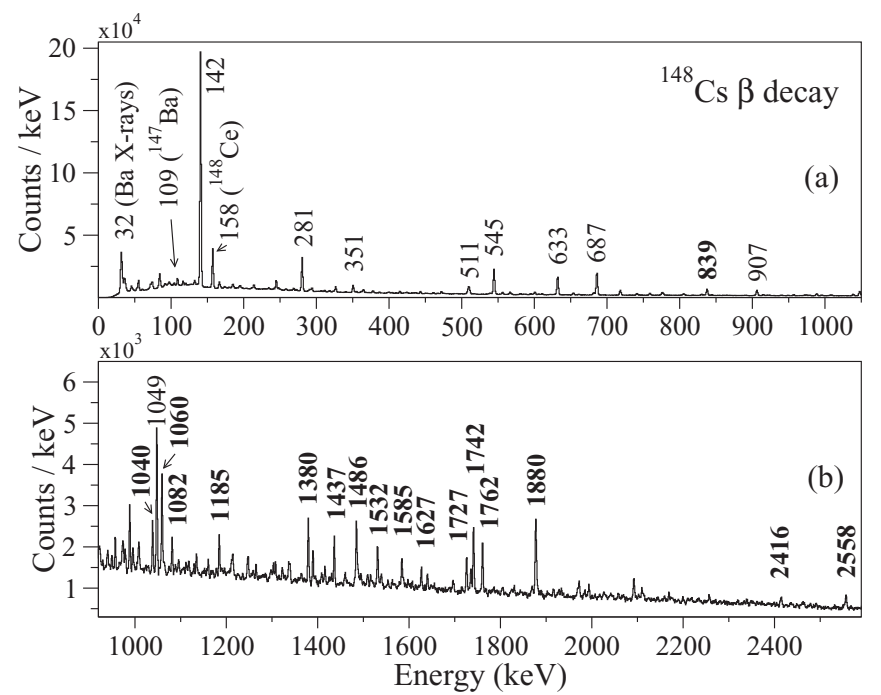

FIG. 1. ${ }^{148} \mathrm{Cs} \rightarrow{ }^{148} \mathrm{Ba} \beta$-gated $\gamma$ spectrum recorded in the first $500 \mathrm{~ms}$ relative to a proton pulse. The main transitions populated through $\beta$ decay in ${ }^{148} \mathrm{Ba}$ are labeled. Newly found transitions are indicated in bold. Contributions by the $\beta-n$ channel and granddaughter decay are indicated.

using a standard TAC (time-to-amplitude converter) electronic module. Further details on the experimental setup and analysis techniques can be found in Ref. [19].

\section{EXPERIMENTAL RESULTS}

\section{A. ${ }^{148} \operatorname{Cs} \beta$ decay}

Figure 1 shows the $\beta$-gated $\gamma$ spectrum corresponding to the decay ${ }^{148} \mathrm{Cs} \rightarrow{ }^{148} \mathrm{Ba}\left(T_{1 / 2}=152(1) \mathrm{ms}\right.$ [19]) recorded in the first $500 \mathrm{~ms}$ after a proton pulse, to remove contributions from long-lived activity. The spectrum shows primarily transitions belonging to this decay, together with contributions from the $\beta$-delayed neutron-emission channel $(\beta-n)$ and the granddaughter decay. Newly measured transitions are indicated in bold.

The $\beta$ decay half-lives of transitions in ${ }^{147} \mathrm{Ba}$, which are expected to be populated as the $\beta$ - $n$ channel, were investigated in order to rule out a possible ${ }^{147} \mathrm{Cs}$ contamination in the incoming beam. The time behavior of the $109.8-\mathrm{keV}$ transition in ${ }^{147} \mathrm{Ba}$ is equivalent to that of the $2^{+} \rightarrow 0^{+} 141.6-\mathrm{keV}$ transition in ${ }^{148} \mathrm{Ba}$. This is shown in Fig. 2, where the two spectra have been normalized for comparison. The half-life extracted from the decay curve of the $109.8-\mathrm{keV}$ line is $T_{1 / 2}=158(6) \mathrm{ms}$, in good agreement with the value for ${ }^{148} \mathrm{Cs}\left(T_{1 / 2}=152(1) \mathrm{ms}\right)$ reported in Ref. [19], while it differs significantly from the adopted one for ${ }^{147} \mathrm{Cs}\left(T_{1 / 2}=230\right.$ (1) $\mathrm{ms}$ [20]).

Examples of prompt $\gamma \gamma$ spectra are shown in Fig. 3: coincidences with the $2^{+} \rightarrow 0^{+}$transition at $141.6 \mathrm{keV}$ are given in panel (a), coincidences with the $4^{+} \rightarrow 2^{+}$transition at $281.3 \mathrm{keV}$ in panel (b), while panel (c) shows coincidences with the $1^{-} \rightarrow 0^{+}$line at $633.1 \mathrm{keV}$.

The low-spin structure of ${ }^{148} \mathrm{Ba}$, already reported in [9,11], was confirmed, and the level scheme of this nucleus was greatly extended, including up to 38 levels and 74 transitions (out of

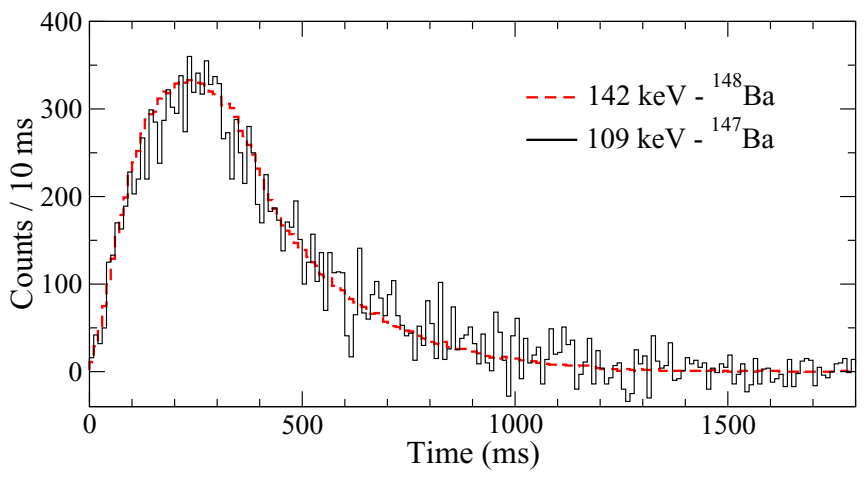

FIG. 2. Time distribution relative to the proton pulse of the 109.8and 141.6-keV transitions from ${ }^{147} \mathrm{Ba}$ and ${ }^{148} \mathrm{Ba}$, respectively. After renormalizing the 141.6-keV time distribution (factor 0.0286), the overlap of the two distributions is in very good agreement with the assumption that levels in ${ }^{147} \mathrm{Ba}$ are only fed through the $\beta$ - $n$ decay of ${ }^{148} \mathrm{Cs}\left(T_{1 / 2}=152(1) \mathrm{ms}\right)$ rather than the $\beta$ decay of ${ }^{147} \mathrm{Cs}\left(T_{1 / 2}=\right.$ 230(1) $\mathrm{ms}$ ).

which 32 levels and 64 transitions are newly observed), as shown in Table I and Fig. 4. The new transitions were identified from coincidences with the previously reported ones, by energy matching and also by analyzing their decay half-lives, when the statistics allowed for it.

The thickness of the lines shown in Fig. 4 scales with the relative intensity of the $\gamma$ transitions, normalized to the one at $141.6 \mathrm{keV}$, as shown in column 5 of Table I. The absolute $\gamma$-ray intensities were determined using, as normalization, the adopted absolute intensities of both daughter and granddaughter isotopes $I_{a b s}\left({ }^{148} \mathrm{La} ; 133.5 \mathrm{keV}\right)=3.9(1) \%$ [21] and $I_{a b s}\left({ }^{148} \mathrm{Ce} ; 158.5 \mathrm{keV}\right)=56(1) \%$ [22], assuming no feeding to the ground state of ${ }^{148} \mathrm{Ce}$ from the ${ }^{148} \mathrm{La}$ decay. For the $141.6-\mathrm{keV}$ transition a theoretical conversion coefficient of $0.574(8)$ has been employed, using BRICC [23], corresponding to a pure E2 multipolarity. The conversion coefficient for

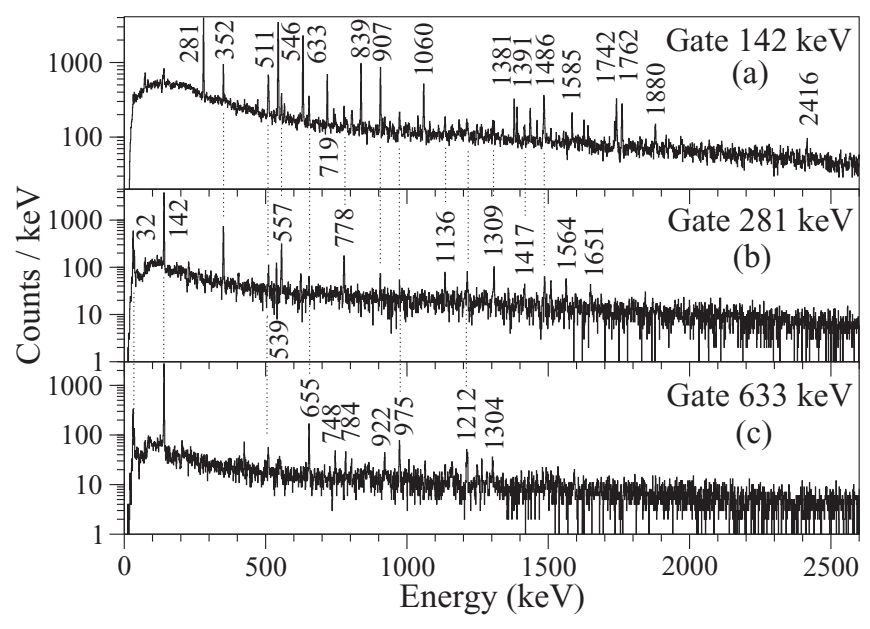

FIG. 3. Spectra extracted from $\gamma-\gamma$ matrices in coincidence with the 141.6-, 281.3-, and 633.1-keV $\gamma$ rays from ${ }^{148} \mathrm{Ba}$ populated in the $\beta$ decay of ${ }^{148} \mathrm{Cs}$ (top to bottom). Coincident transitions are indicated by their energy. 
TABLE I. Levels populated in the decay ${ }^{148} \mathrm{Cs} \rightarrow{ }^{148} \mathrm{Ba}$. The first column from the left reports level energies in keV and the second proposed spins and parities. Columns 3 and 4 show the $I_{\beta}$ calculated as discussed in the text and corresponding $\log (f t)$ values. In the last columns we report the energy of the $\gamma$ transition deexciting each level, together with its relative intensity (normalized to the $2^{+} \rightarrow 0^{+}$transition) and the level to which it decays. Previously known levels and transitions are marked by $\left(^{*}\right)$. For absolute intensity per $100 \beta$ decays, multiply the $\gamma$ intensities by 0.30(4). Favored spin assignments are indicated in bold, as discussed in Sec. IIIC.

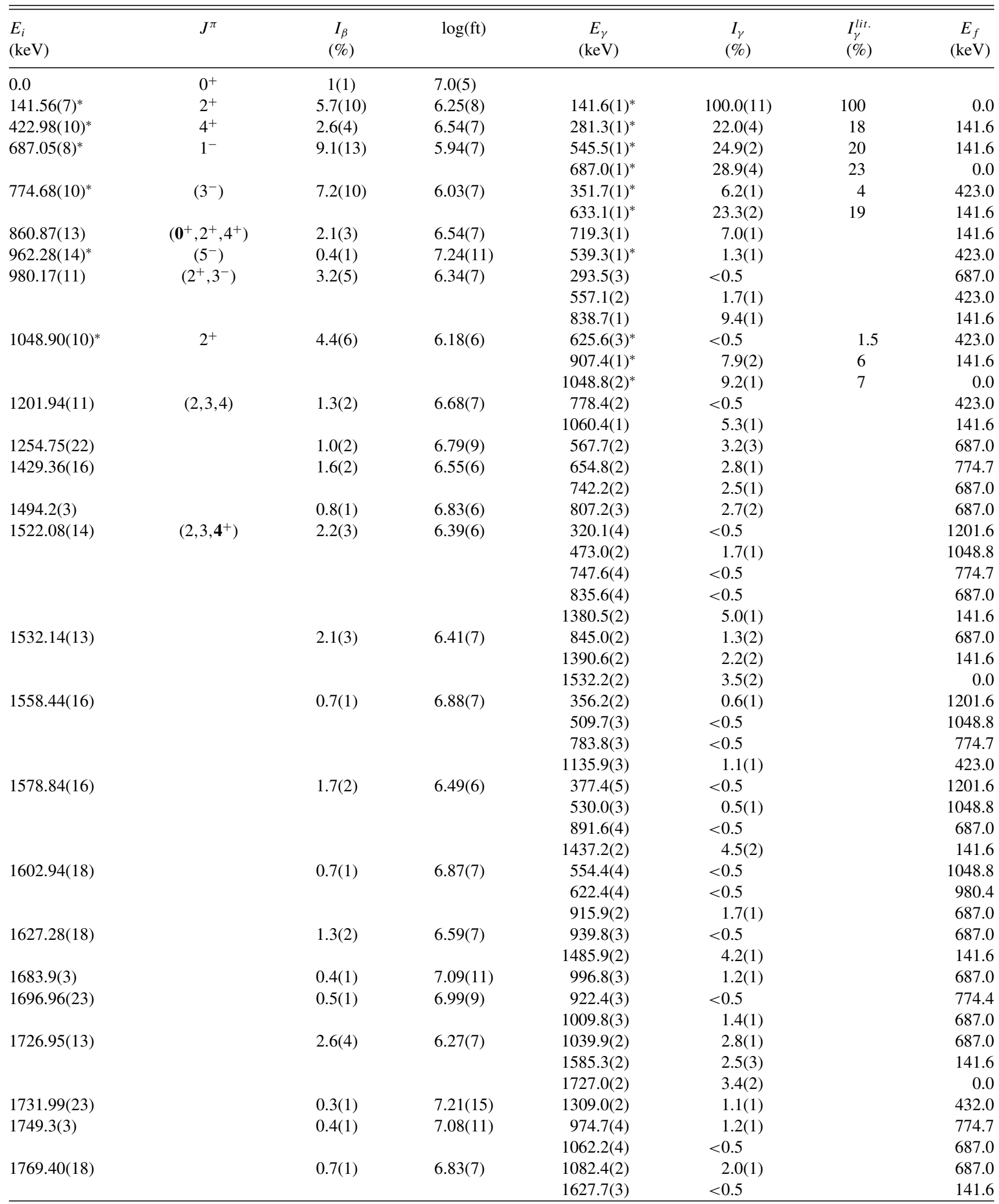


TABLE I. (Continued.)

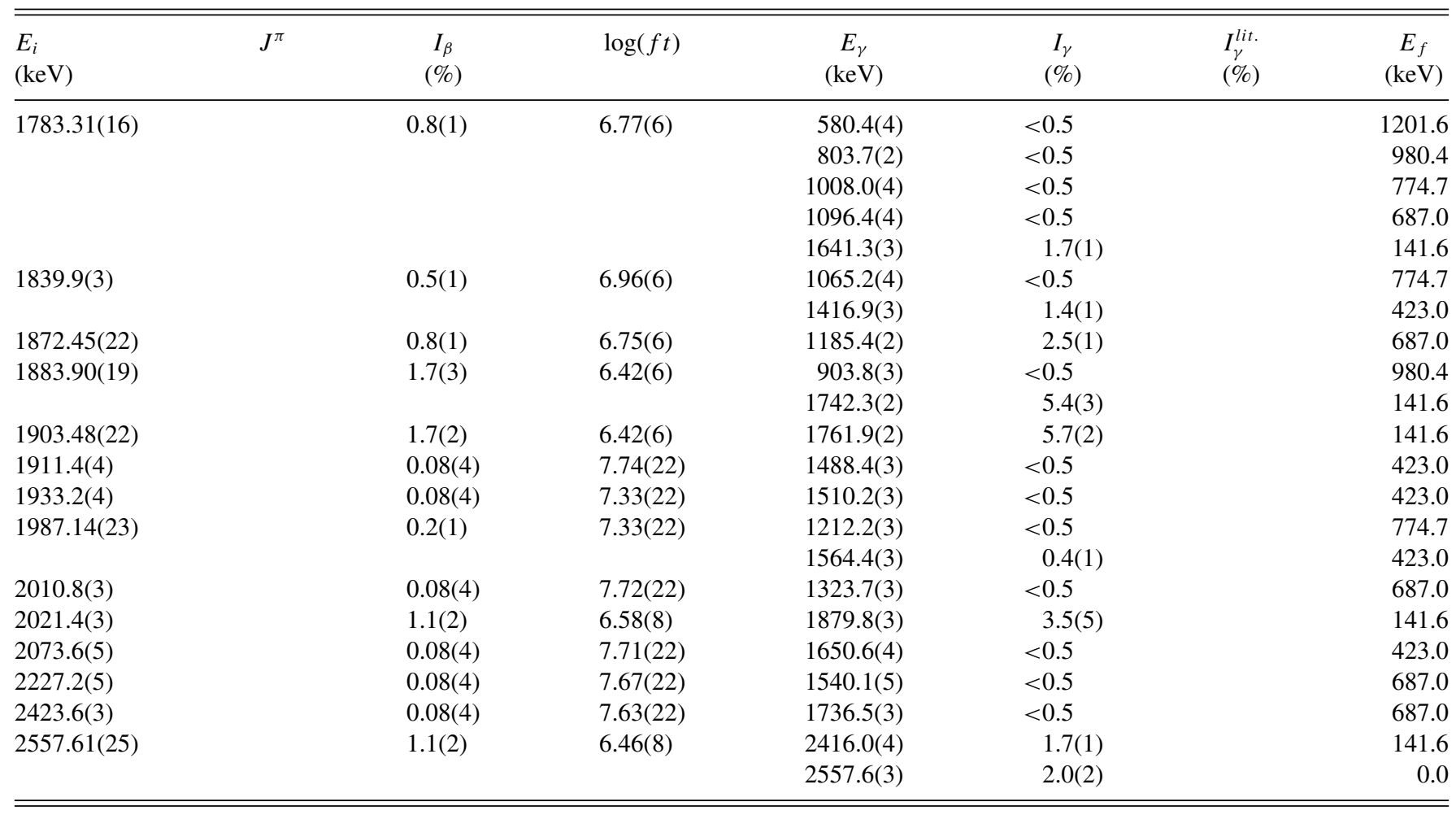

higher energy transitions is small and was neglected in the further analysis.

The absolute $\beta$ feeding contributions corresponding to each level were extracted by taking into account the feeding and depopulating transitions. The $I_{\beta}$ towards the ground state were estimated from the missing feeding, once the intensity going to delayed neutron emission was accounted for (subtracted). They are reported in column 3 of Table I and in the left side of the level scheme in Fig. 4. Using the calculated half-life and a $Q_{\beta}$ of $10.3(6) \mathrm{MeV}$ [24], the corresponding $\log (f t)$ values were extracted, as shown in column 4 of Table. I.

The ${ }^{148} \mathrm{Cs}$ neutron emission probability of $P_{n}=38(4) \%$ was determined from the ratio between the total number of ${ }^{147} \mathrm{Ba}$ and ${ }^{148} \mathrm{Ba}$ nuclei produced, estimated using the adopted values $I_{a b s}\left({ }^{147} \mathrm{La} ; 167.4 \mathrm{keV}\right)=15.3(16) \%$ and $I_{a b s}\left({ }^{147} \mathrm{La} ; 196.1 \mathrm{keV}\right)=6.7(7) \%[25]$.

\section{B. ${ }^{150} \mathrm{Cs} \beta$ decay}

Transitions belonging to the decay ${ }^{150} \mathrm{Cs} \rightarrow{ }^{150} \mathrm{Ba}$ were studied for the first time: Fig. 5(a) shows the spectrum following the $\beta$ decay of ${ }^{150} \mathrm{Cs}$, extracted by closing the beam gate after $200 \mathrm{~ms}$ and subtracting the long-lived activity. Intense short-lived transitions at 101.1, 217.1, 597.4, 613.4, and $945.7 \mathrm{keV}$ are associated with the internal deexcitation of ${ }^{150} \mathrm{Ba}$. The lines marked with asterisks belong to the $\beta-n$ channel being observed in the decay scheme of ${ }^{149} \mathrm{Ba}$ [19]. All these lines correspond to the same decay half-life of 80(3) ms. An additional strong transition is seen in the spectrum, marked by the " $\$$ " symbol: this is coming from the decay ${ }^{149} \mathrm{Ba} \rightarrow{ }^{149} \mathrm{La}$, identified as originating from the $\beta-n$ decay branch. Since the presence of contaminant species in the beams has been carefully checked, the presence of transitions belonging to ${ }^{149} \mathrm{Ba}$ in the decay spectrum of ${ }^{150} \mathrm{Cs}$ can be traced back to the $\beta$-delayed neutron emission channel.

As a comparison, the spectrum associated with the longlived activity is shown in panel (b), where known lines, belonging mainly to the decay of ${ }^{150} \mathrm{Pm}$, are visible.

Panels (c), (d), (e), and (f) show $\beta \gamma \gamma$ coincidence spectra obtained by gating on the transitions indicated in each panel. Coincident peaks originating from the Compton scattering of the strong background 511-keV $\gamma$ activity are visible, and are indicated in the spectra by the " $\&$ " symbol.

The analysis of $\gamma \gamma$ coincidences provided the level scheme shown in Fig. 6: the thickness of each arrows is proportional to its intensity, normalized to the $2^{+} \rightarrow 0^{+}$transition, as reported in column 5 of Table II. The transition at $975.9 \mathrm{keV}$ is tentative, and is indicated by a dashed line since it is only seen in coincidence with the 101.1-keV $\gamma$ line.

The absolute $\gamma$-ray intensities were determined using, as normalization, the absolute intensities $I_{a b s}(97.0 \mathrm{keV})=$ $30 \%$ and $I_{a b s}(208.7 \mathrm{keV})=25 \%$ [26] from the granddaughter ${ }^{150} \mathrm{Ce}$, assuming no feeding to the ground state from the ${ }^{150} \mathrm{La}$ decay. For the transition at $101.1 \mathrm{keV}$ a conversion coefficient of 1.86(3) [23] has been used, assuming a pure E2 character. Coefficients for the higher energy transitions are small and have been neglected.

Even if a strong contribution from the $\beta$-delayed neutron emission branch is seen in the spectrum, the low statistics of 


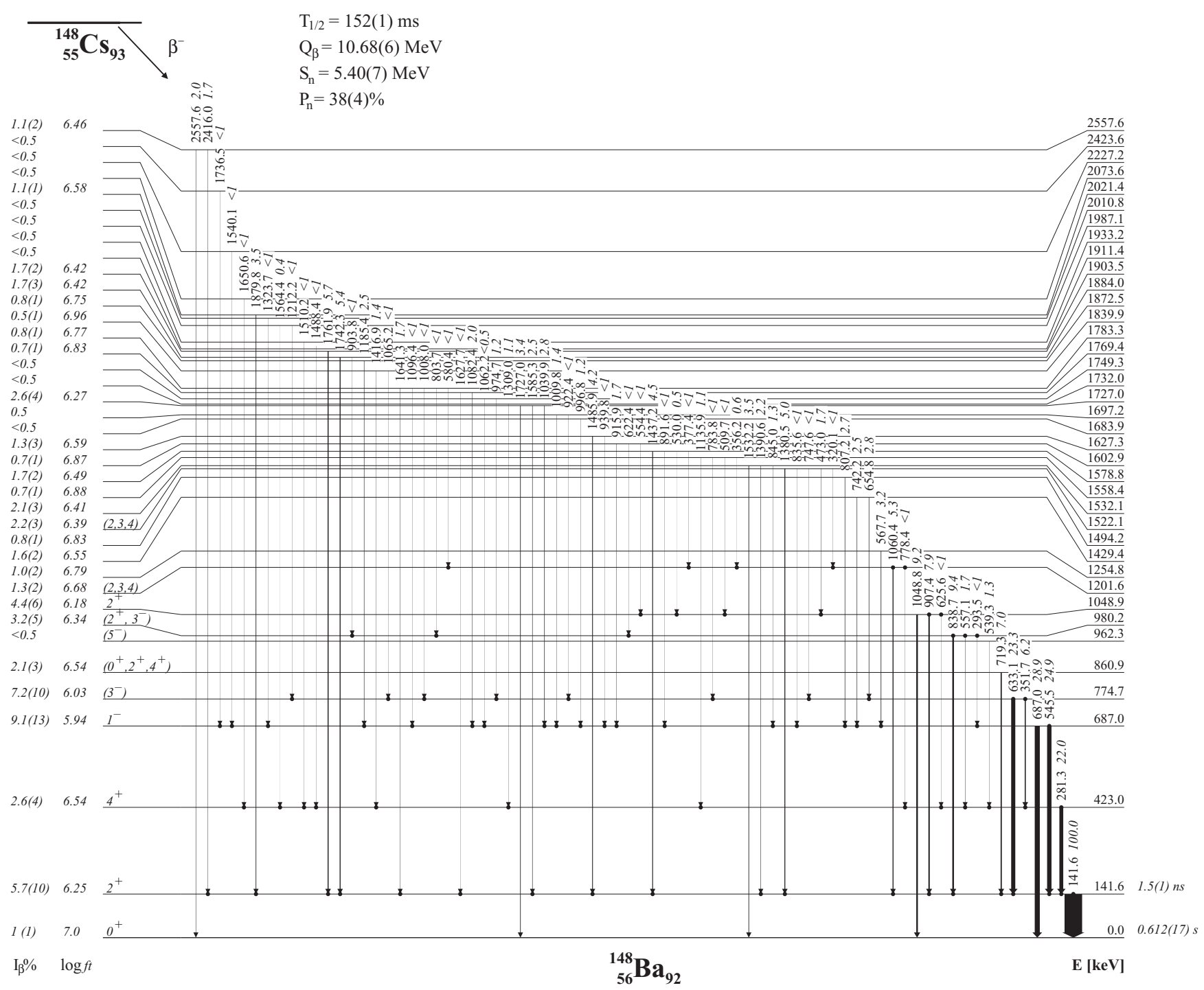

FIG. 4. Extended level scheme for the decay ${ }^{148} \mathrm{Cs} \rightarrow{ }^{148}$ Ba extracted from $\gamma \gamma$ coincidences. The $I_{\beta}$ and $\log f t$ are calculated from intensity balance as discussed in the text. For absolute intensity per $100 \beta$ decays, multiply the $\gamma$ intensities by $0.30(4)$.

the data and absence of information on the granddaughters populated by the two channels did not allow for a precise estimation of the $P_{n}$ value. The ratio of intensities of the most populated transitions in ${ }^{149} \mathrm{Ba}$ [19] and ${ }^{150} \mathrm{Ba}$ nuclei, 316.6and 101.1-keV transitions, respectively, of the order of $44 \%$, suggests a steep increase in the $\beta$-delayed neutron emission probability in these exotic members of the Cs chain. This is in agreement with state-of-the-art calculations $[27,28]$ which predict $P_{n}$ to be $41 \%$. We therefore use this theoretical value for the evaluation of the $\beta$ feeding to the states, reported in Table II and Fig. 6.

The absolute $\beta$ feeding corresponding to each state was extracted using the procedure described earlier for ${ }^{148} \mathrm{Cs}$ decay. Given the large $Q_{\beta}$ window, the extracted $I_{\beta}$ have to be considered as upper limits.

Table II reports the energy, proposed $J^{\pi}$ assignments (which will be discussed later in Sec. IIIC), de-populating $\gamma$ transitions, and relative intensities of the levels.

\section{Spin assignments}

The level scheme of ${ }^{148} \mathrm{Ba}$ has been greatly extended. Starting from the known low-spin level structure, together with considerations involving the decay patterns and systematics, spins and parities of several levels are proposed:

(i) The $860.9-\mathrm{keV}$ level is only decaying to the $2_{1}^{+}$via the $719.9-\mathrm{keV}$ transition. No feeding transition to this state is seen. Although other assignments are possible, as indicated in Table I, this state is interpreted as $0_{2}^{+}$, given the close resemblance to a similar state found in ${ }^{146} \mathrm{Ba}$ in Ref. [14].

(ii) The $980.2-\mathrm{keV}$ level decays to $1_{1}^{-}$state through the $293.5-\mathrm{keV}$ transition, to the $4_{1}^{+}$via the $557-\mathrm{keV}$ one, and to the $2_{1}^{+}$via the $839.6-\mathrm{keV} \gamma$ ray. This decay pattern closely resembles that of the $J^{\pi}=3^{-}$state, even if the $I_{\beta}$ is similar to the one measured for the 


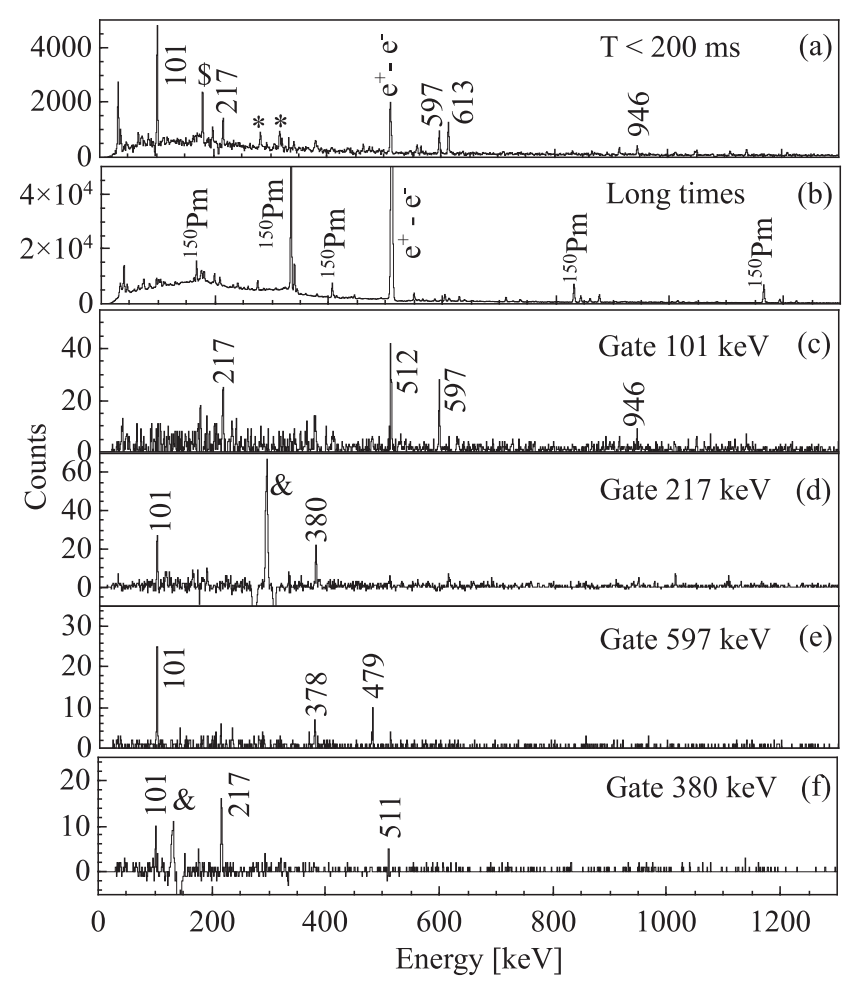

FIG. 5. (a) ${ }^{150} \mathrm{Cs}$ decay spectrum acquired within $200 \mathrm{~ms}$ from the proton pulse impinging on the UCx target. Long-lived activity (b) has been subtracted. Lines belonging to daughter decay are marked by their energy, while asterisks indicate lines coming from the $\beta$-delayed neutron emission channel (i.e. ${ }^{149} \mathrm{Ba}$ ) and its subsequent decay (marked by the dollar symbol). Panels (c), (d), (e) and (f) show spectra in coincidence with transitions at 101.1, 217.1, 597.4 and $380.2 \mathrm{keV}$, respectively. The presence of peaks arising from Compton scattering of the $511-\mathrm{keV} \gamma$ rays is indicated by "\&" symbol.

other two $J^{\pi}=2^{+}$states, which have been established in previous works.

(iii) The state at $1202 \mathrm{keV}$ shows decays towards the $4_{1}^{+}$ and $2_{1}^{+}$states, pointing to a $J$ assignment of 3 .

(iv) The 1522-keV level, decaying with the 473-keV line to the $2_{2}^{+}$, and with the $1381-\mathrm{keV}$ transition to $2_{1}^{+}$one, is a possible candidate for the $4_{2}^{+}$. Its $\log f t=6.39(6)$ is also comparable to the one measured for $4_{1}^{+}[6.54(7)]$. We therefore favou the $J^{\pi}=4^{+}$assignment.

We do not see high- $J$ states, apart from the $5^{-}$level at $962.3 \mathrm{keV}$ [9], which is populated from high-lying states and not directly fed by $\beta$ decay. Ground state feeding seems to be very weak in ${ }^{148} \mathrm{Ba}$ compared to the value reported for ${ }^{146} \mathrm{Ba}$ $\left(I_{\beta}=27 \%\right)$ [14].

The level scheme of ${ }^{150} \mathrm{Ba}$ is extracted for the first time and, based on the coincidences shown and on systematic comparison with the lighter members of Ba chain, we associate the level at $101.1 \mathrm{keV}$ to the $2_{1}^{+}$state, the level at $318.2 \mathrm{keV}$ to the $4_{1}^{+}$, while the level at $613.4 \mathrm{keV}$ is proposed as the $1_{1}^{-}$level and the one at $698.5 \mathrm{keV}$ as the $3_{1}^{-}$state. The decay pattern of these states closely resembles that of ${ }^{148} \mathrm{Ba}$ : the $\beta$ decay seems to preferentially populate the $0^{+}, 2^{+}, 1^{-}$, and $3^{-}$states in both
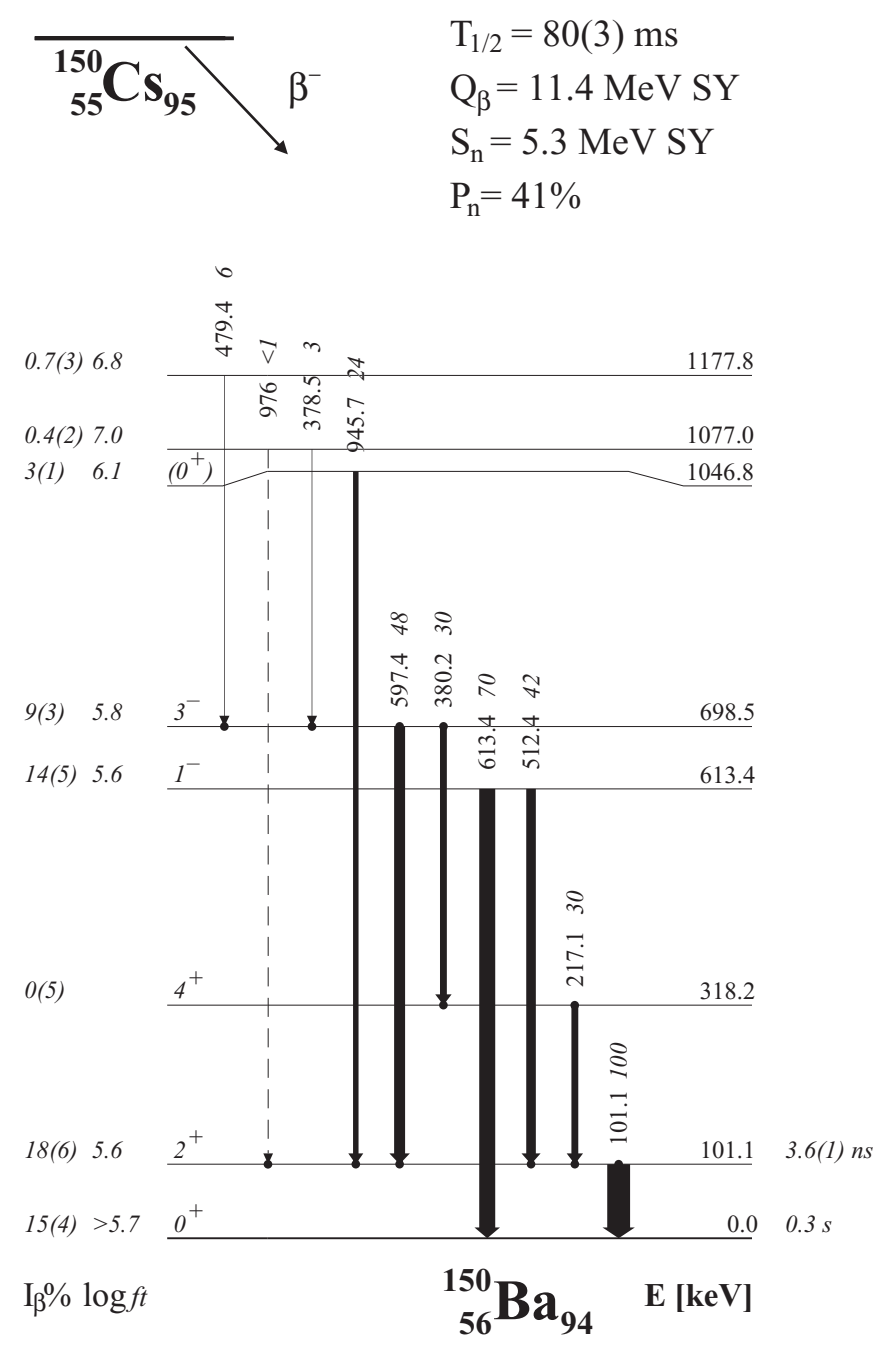

FIG. 6. Proposed scheme for the decay ${ }^{150} \mathrm{Cs} \rightarrow{ }^{150} \mathrm{Ba}$ extracted from $\beta \gamma \gamma$ coincidences. The $Q_{\beta}$ value is taken from systematics [24], while the $I_{\beta}$ and $\log f t$ are calculated from intensity balance as discussed in the text. For absolute intensity per $100 \beta$ decays, multiply the $\gamma$ intensities by $0.12(4)$. The indicated $P_{n}$ value is taken from [27].

isotopes. At variance from ${ }^{148} \mathrm{Ba}$, no feeding to the $4^{+}$state has been obtained.

The analysis of the population pattern and a comparison with neighboring nuclei suggest that the state at $1046.8 \mathrm{keV}$ could be the $0_{2}^{+}$. No firm assignment can be established for the levels at 1077 and $1178 \mathrm{keV}$. The systematics of the levels found in the lightest members of the isotopic chain seem to point to a $J^{\pi}=2^{+}$assignment for the first level, but its decay, showing a strong branch towards the $3^{-}$state and a weaker branch populating the $2^{+}$state, does not fully confirm this hypothesis.

The $\log (f t)$ deduced from the $\beta$ feeding indicate a competition between Gamow-Teller (GT) and first-forbidden (ff) transitions, since both positive- and negative-parity states seem to be populated with similar intensities. This is a typical pattern in the $\mathrm{Ba}$ chain, observed also in the case of ${ }^{146} \mathrm{Ba}$. We stress that a strong contribution from the Pandemonium effect is expected, given the large $Q_{\beta}$ window of the decays under study. 
TABLE II. Levels populated in the decay ${ }^{150} \mathrm{Cs} \rightarrow{ }^{150} \mathrm{Ba}$. The first column from the left reports level energies in $\mathrm{keV}$ and the second proposed spins and parities. Columns 3 and 4 show the $I_{\beta}$ calculated as discussed in the text and corresponding $\log (f t)$ values. In the last columns we report the energies of the $\gamma$ transitions de-exciting each level, together with their relative intensities and the level towards which it decays. For absolute intensity per $100 \beta$ decays, multiply the $\gamma$ intensities by $0.12(4)$. Owing to the large $Q_{\beta}$ window, the extracted $I_{\beta}$ have to be considered as upper limits, and the $\log (f t)$ as lower limits, as indicated in Fig. 6. The transition reported in italics is tentative since it is only seen in coincidence with the $101.1-\mathrm{keV} \gamma$ line.

\begin{tabular}{lccccrr}
\hline \hline $\begin{array}{l}E_{i} \\
(\mathrm{keV})\end{array}$ & $J^{\pi}$ & $\begin{array}{c}I_{\beta} \\
(\%)\end{array}$ & $\log (f t)$ & $\begin{array}{c}E_{\gamma} \\
(\mathrm{keV})\end{array}$ & $\begin{array}{c}I_{\gamma} \\
(\%)\end{array}$ & \multicolumn{1}{c}{$\begin{array}{c}E_{f} \\
(\mathrm{keV})\end{array}$} \\
\hline 0.0 & $0^{+}$ & $<15(4)$ & $>5.7$ & & & \\
$101.1(1)$ & $\left(2^{+}\right)$ & $18(6)$ & 5.6 & $101.1(1)$ & $100(6)$ & 0.0 \\
$318.3(2)$ & $\left(4^{+}\right)$ & $0(5)$ & & $217.1(2)$ & $30(4)$ & 101.1 \\
$613.8(1)$ & $\left(1^{-}\right)$ & $14(5)$ & 5.6 & $512.4(3)$ & $42(7)$ & 101.1 \\
& & & & $613.8(1)$ & $70(4)$ & 0.0 \\
$698.5(2)$ & $\left(3^{-}\right)$ & $9(3)$ & 5.8 & $380.2(3)$ & $30(4)$ & 318.2 \\
& & & & $597.4(2)$ & $48(4)$ & 101.1 \\
$1046.8(2)$ & $\left(0^{+}, 2^{+}, 4^{+}\right)$ & $3(11)$ & 6.1 & $945.7(2)$ & $24(3)$ & 101.1 \\
$1077.0(3)$ & & $0.4(2)$ & 7.1 & $378.5(4)$ & $3(1)$ & 698.5 \\
& & & & $975.9(3)$ & $<1$ & 101.1 \\
$1177.9(4)$ & & $0.7(3)$ & 6.9 & $479.4(3)$ & $6(1)$ & 698.5 \\
\hline \hline
\end{tabular}

In Fig. 7 a comparison of low-lying states in ${ }^{146,148,150} \mathrm{Ba}$ is given. Newly observed transitions and newly proposed spins and parities are marked with italics red labels. The levels are marked by the proposed $J^{\pi}$ here discussed.

\section{Fast-timing measurements}

The statistics collected in the two data sets allowed to study the lifetime of the $2_{1}^{+}$states in both ${ }^{148} \mathrm{Ba}$ and ${ }^{150} \mathrm{Ba} . E_{\gamma_{1}}-E_{\gamma_{2}}$ time triple coincidence matrices have been constructed within a coincidence-time window of $50 \mathrm{~ns}$, making use of all possible combinations of $\operatorname{LaBr}_{3}(\mathrm{Ce})$ detectors, with the additional request of a registered $\beta$ decay.

The time spectra are obtained by projecting on the time axis the region around the peaks [indicated by the circular polygon in the matrix shown in Fig. 8 (a)]. The lifetime of the level is constructed using feeding transitions as start transitions
( $Y$ axis) and the decay-out transition as stop transition ( $X$ axis). In the specific case the chosen feeding transitions are those at $281-545-633 \mathrm{keV}$ while the deexciting transition is the 141.6$\mathrm{keV} 2^{+} \rightarrow 0^{+}$transition.

An accurate subtraction of the background is obtained by considering a two-dimensional gate in the energy plane, around the corresponding coincidence peak, as shown in the same panel for the 281-, 546-, and 633-keV transitions. This procedure allows for a better control of contaminant contributions which may have different time characteristics than the average background, and reduces the statistical uncertainty associated with background subtraction by considering a wider two-dimensional background region. The resulting spectrum is shown in panel (c), together with the fit consisting of the convolution between a gaussian and an exponential function. The resulting half-life is also indicated in the panel.

Low statistics did not allow for a similar procedure in the case of ${ }^{150} \mathrm{Ba}$ and the half-life is determined using a twodimensional $E_{\gamma}$-time matrix. The time information is extracted from the $\beta-\mathrm{LaBr}_{3}(\mathrm{Ce}) \mathrm{TAC}$. Contributions from long-lived daughter activity relative to the proton impact have been subtracted. From this matrix one can also judge the quality of the walk correction applied, since the shape of the time distribution does not change significantly going towards low energies.

The time response of the $2^{+} \rightarrow 0^{+}$transition is shown in panel (d). The exponential fit did not include the first 800 ps corresponding to prompt transitions. No contribution from high-lying levels is expected, as it should mainly come from the $4^{+}$state, which is not fed in $\beta$ decay. Moreover, this state is expected to show a shorter half-life, of the order of tens of ps, which does not affect our result.

The half-lives extracted for the two nuclei, 1.5(1) ns for ${ }^{148} \mathrm{Ba}$ and 3.4(2) ns for ${ }^{150} \mathrm{Ba}$ are longer than those extracted for ${ }^{142-146} \mathrm{Ba}$, of the order of few hundreds ps [29]. This is expected given the lower transition energy, and points towards an increasing collectivity. These results can be translated into $B\left(E 2: 2^{+} \rightarrow 0^{+}\right)$of $90.4(25)$ and 110(4) W.u., for ${ }^{148,150} \mathrm{Ba}$, respectively.

\section{DISCUSSION}

A qualitative description of the evolution of the deformation, at least in the quadrupole degree of freedom, can be given
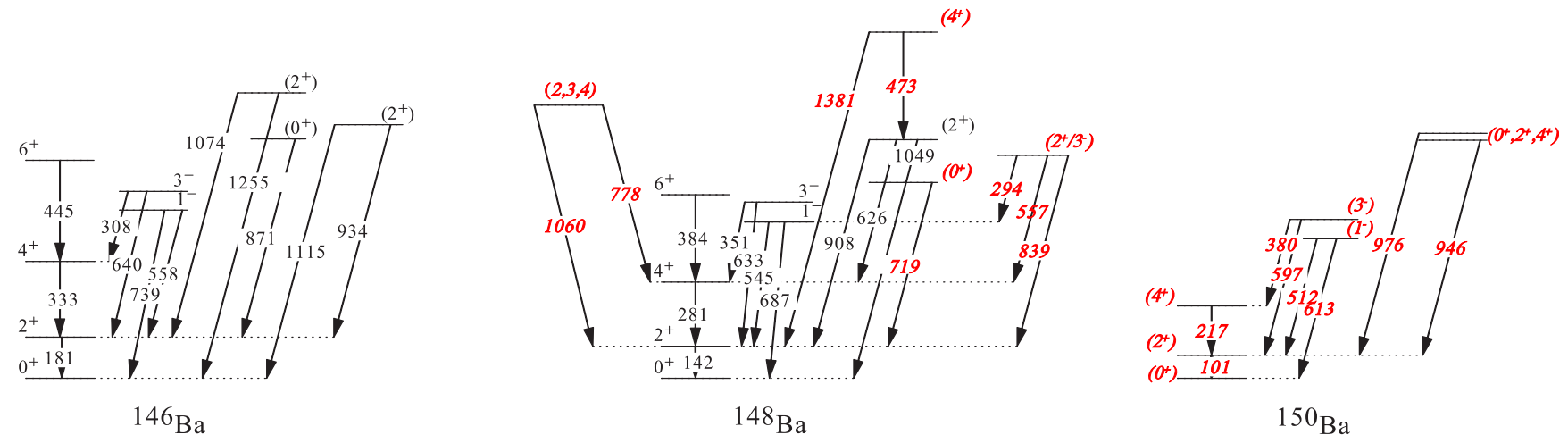

FIG. 7. Comparison between observed low-lying states and corresponding decaying transitions in ${ }^{146,148,150}$ Ba. Transitions reported in this work for the first time and newly proposed $J^{\pi}$ assignments are highlighted in red italics. Levels and transitions of ${ }^{146} \mathrm{Ba}$ are taken from Ref. [14]. 


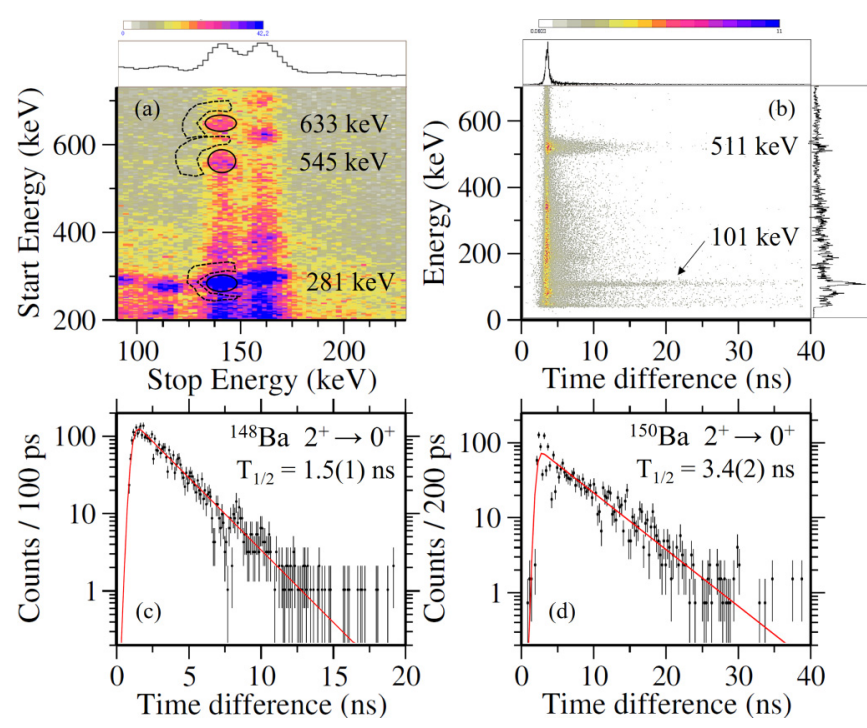

FIG. 8. (a) Two-dimensional projection on the energy plane of a $\gamma \gamma$ TAC coincidence matrix built for ${ }^{148} \mathrm{Ba}$. The black circular regions indicate the location of the bidimensional gates applied to extract the spectrum in panel (c) and the dotted shapes represent the background region which was subtracted. The time response obtained for the $2^{+} \rightarrow 0^{+}$transition in ${ }^{148} \mathrm{Ba}$ is shown, together with the convolution fit in panel (c). Panel (b) shows the $\beta$-TAC spectrum constructed for ${ }^{150} \mathrm{Ba}$, while its projection is shown in panel $(\mathrm{d})$. More details are presented in the text.

in terms of phenomenological quantities such as the ratio of the energies $E\left(4_{1}^{+}\right) / E\left(2_{1}^{+}\right)$, which results in a value of 3.1 , very close to the axially symmetric rotor limit [30].

Based on the proposed spins, we can further study quantities which can give a hint onto the degree of triaxiality. The ratio between the energies of the $2_{2}^{+}$and those of the $4_{1}^{+}$and $2_{1}^{+}$states, calculated following the relation $E_{S} / E\left(2_{1}^{+}\right)=\frac{E\left(2_{2}^{+}\right)-E\left(4_{1}^{+}\right)}{E\left(2_{1}^{+}\right)}$, can discriminate between $\gamma$-unstable $\left[E_{S} / E\left(2_{1}^{+}\right)=0\right]$ and $\gamma$-rigid $\left[E_{S} / E\left(2_{1}^{+}\right)<0\right]$ shapes [31,32]. The large positive value found for ${ }^{148} \mathrm{Ba}$, the highest in the $\mathrm{Ba}$ isotopic chain, points again to the evolution of more axially symmetric shapes.

The experimental data shown here have been interpreted in the framework of SCCM calculations based on the Gogny D1S energy density functional [33]. In the calculations the particle number, parity, and angular momentum projections are carried out, and axial quadrupole and octupole shapes are allowed to mix within the generator coordinate method (GCM). Therefore, nuclear states are defined as linear combinations of projected mean-field states with different quadrupole and octupole deformations. The most favorable deformations for each state are found self-consistently by minimising the GCM energy [16].

This approach has been recently successfully applied to describe the new results coming from the CARIBU experiments on ${ }^{144-146} \mathrm{Ba}[13,16]$. In this paper we report calculations performed in the $\mathrm{Ba}$ isotopic chain ranging from $N=86$ to $N=94$ in order to track the evolution of deformation.

Potential energy surfaces (PESs) as a function of the axial quadrupole $\left(\beta_{2}\right)$ and octupole $\left(\beta_{3}\right)$ deformation parameters for the nuclei ${ }^{142-150} \mathrm{Ba}$, defined as in Ref. [16], are shown in Fig. 9. The energy is symmetric under reflection about the $\beta_{3}=0$ axis since the interaction conserves parity. The absolute minima in all of the isotopes are found in the prolate part $\left(\beta_{2}>0\right)$, and the position of the minima is displaced to larger deformations with increasing the number of neutrons in the system $\left(\beta_{2} \sim 0.2\right.$ and $\sim 0.3$ in ${ }^{142} \mathrm{Ba}$ and ${ }^{150} \mathrm{Ba}$, respectively). In addition, the evolution of a secondary minimum in the oblate side (and $\beta_{3}=$ $0)$ is also obtained, which is evolving and moving away from
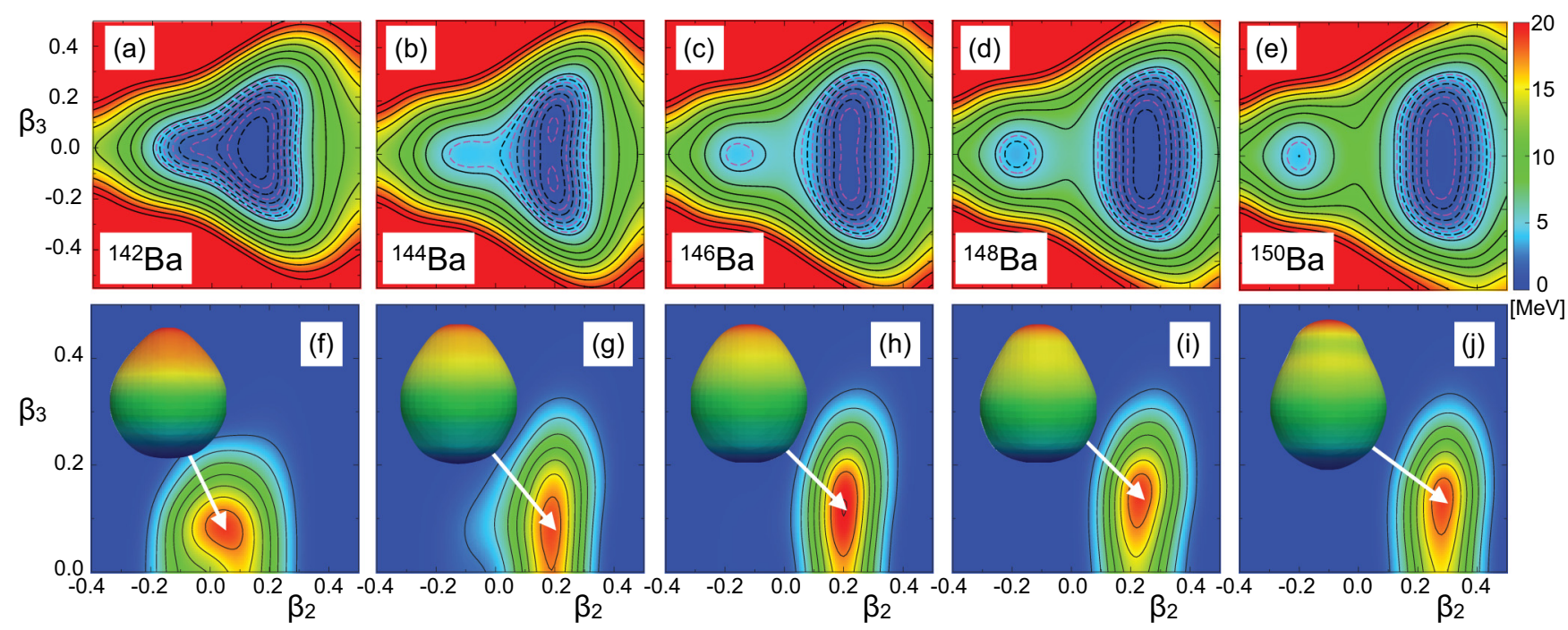

FIG. 9. (a)-(e) Potential energy surfaces in the $\left(\beta_{2}, \beta_{3}\right)$ plane for the Ba isotopic chain for $N=86-94$. Deformed minima, both prolate and oblate, are seen in all the isotopes, evolving towards larger deformations in the heaviest members of the chain. The energy is symmetric about the $\beta_{3}$ axis. See text for details. (f)-(j) Calculated collective wave functions of the ground states $\left(0^{+}\right)$are represented for $\beta_{3} \geqslant 0$. To better visualize the shape of the isotopes, the surface that joins the points with constant spatial density is also shown for each isotope. These densities are computed with the HFB wave functions that correspond to the maximum of each collective wave function (see the arrows). Pear shapes are clearly seen, together with an evolution towards more elongated quadrupole deformations for ${ }^{148-150} \mathrm{Ba}$. 

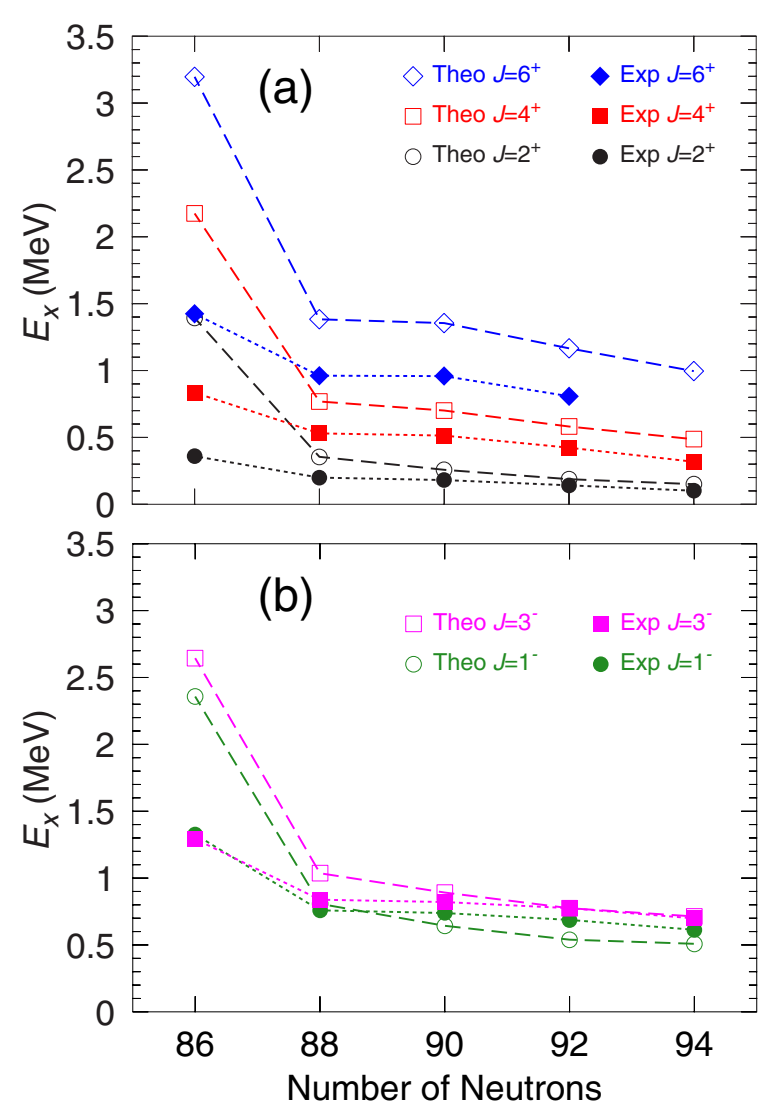

FIG. 10. Systematic comparison of experimental (filled symbols) and theoretical (open symbols) energy spectra in the Ba isotopic chain. Positive-parity states are grouped in panel (a) while the negative-parity ones are grouped in panel (b). Levels in ${ }^{142-148} \mathrm{Ba}$ are taken from the literature [9].

the prolate one in the heavier isotopes. For the nuclei ${ }^{142148} \mathrm{Ba}$ the absolute minima correspond to octupole deformed $\left(\beta_{3} \neq 0\right)$ intrinsic states, although the energy wells around these minima are rather soft in the $\beta_{3}$ direction. This holds also for ${ }^{150} \mathrm{Ba}$ where the minimum is at $\beta_{3}=0$. This is a clear indication that beyond-mean-field correlations should be taken into account, i.e., the restoration of the symmetries broken at the HFB level (particle number, parity, and angular momentum) and the shape mixing (quadrupole and octupole). After performing such symmetry restorations and shape mixing, we obtain both the spectra (excitation energies and electromagnetic moments and transition probabilities) and the collective wave functions (CWFs). These CWFs represent the weights of the different shapes in the different nuclear states.

We can then compare the systematics of the excitation energies of the lowest positive- and negative-parity bands obtained with the present SCCM method. In Fig. 10 we show the theoretical results and the experimental data that include the newly identified excitation energies corresponding to ${ }^{150} \mathrm{Ba}$ levels. The qualitative behavior of the experimental results is nicely reproduced along the isotopic chain. However, the theory predicts a sharper transition (both in positive- and negative-parity states) from an almost spherical nucleus $\left({ }^{142} \mathrm{Ba}\right)$ to quadrupole-deformed ones $\left({ }^{144-150} \mathrm{Ba}\right)$. The

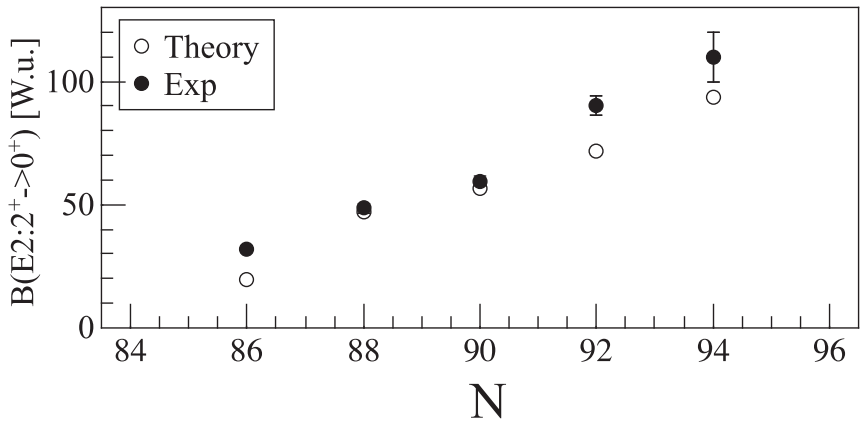

FIG. 11. Filled symbols show $B\left(E 2: 2^{+} \rightarrow 0^{+}\right)$in Ba isotopic chain. They are extracted from the measured lifetimes in the case of ${ }^{148-150} \mathrm{Ba}$ and from Ref. [12,13,29] in the lighter isotopes. The experimental data points are compared to results obtained from the calculations, shown with open symbols.

evolution towards larger quadrupole deformations is seen in the lowering of the energies towards mass 150 . It has to be noticed that the theoretical spectra are systematically stretched in the positive-parity band. This is a well-known effect in this kind of calculations and it can be solved if triaxial and time-reversal symmetry broken states (cranking states) are included in this framework [34].

The increase in the quadrupole collectivity is also observed in the $B\left(E 2: 2^{+} \rightarrow 0^{+}\right)$values along the isotopic chain. In Fig. 11 we show a comparison between the theoretical predictions and the experimental data, where we have added the $B(E 2)$ values extracted from the newly measured lifetimes for ${ }^{148-150} \mathrm{Ba}$. The experimental trend is well reproduced by the present calculations, although theory predicts smaller values for ${ }^{142} \mathrm{Ba}$ and ${ }^{148-150} \mathrm{Ba}$.

Finally, we study the interplay between quadrupole and octupole deformations in these nuclei by analyzing the ground state CWFs obtained with the present SCCM calculations. These CWFs are shown in Figs. 9(f)-9(j), where only the $\beta_{3} \geqslant 0$ quadrant is plotted because they are symmetric under a reflection about the $\beta_{3}=0$ axis. The distributions are all peaked at values of $\beta_{3}$ different from zero and not larger than $\sim 0.2 .{ }^{142} \mathrm{Ba}$ being almost spherical, the rest of the nuclei show a definite quadrupole deformation, which becomes more stable with increasing number of neutrons, with a $\beta_{2}$ parameter around 0.3 in ${ }^{150} \mathrm{Ba}$. We observe that the ground state CWFs are peaked at the minimum of their corresponding potential well given in Figs. 9(a)-9(e). In each panel the isosurface of the spatial density $\left(0.08 \mathrm{fm}^{-3}\right)$ is shown, in order to better visualise the shape of the isotopes. These densities are computed with the HFB wave functions that correspond to the maximum of each $\mathrm{CWF}$, indicated by the arrows. Pear shapes are clearly seen, ${ }^{142} \mathrm{Ba}$ being more spherical and ${ }^{150} \mathrm{Ba}$ more quadrupole deformed. To analyze more quantitatively these results, the mean values and fluctuations of the ground state quadrupole and octupole deformations are shown in Table III. Here we observe that the spreading in the $\beta_{3}$ direction is larger than in the quadrupole. Therefore, SCCM calculations predict both well-established quadrupole and soft octupole deformation in ${ }^{144-150} \mathrm{Ba}$ ground states. 
TABLE III. Mean values and fluctuations of the axial quadrupole $\left(\beta_{2}\right)$ and octupole $\left(\beta_{3}\right)$ deformation parameters calculated for the SCCM ground states given in Figs. 9(f)-9(j).

\begin{tabular}{lcccc}
\hline \hline Isotope & $\overline{\beta_{2}}$ & $\overline{\Delta \beta_{2}}$ & $\overline{\beta_{3}}$ & $\overline{\Delta \beta_{3}}$ \\
\hline${ }^{142} \mathrm{Ba}$ & 0.05 & 0.11 & 0.10 & 0.07 \\
${ }^{144} \mathrm{Ba}$ & 0.17 & 0.10 & 0.13 & 0.09 \\
${ }^{146} \mathrm{Ba}$ & 0.20 & 0.07 & 0.14 & 0.09 \\
${ }^{148} \mathrm{Ba}$ & 0.24 & 0.06 & 0.15 & 0.09 \\
${ }^{150} \mathrm{Ba}$ & 0.28 & 0.06 & 0.14 & 0.09 \\
\hline \hline
\end{tabular}

These results are consistent with the small excitation energies for the $3^{-}$states in ${ }^{142-150} \mathrm{Ba}$ and the large $B(E 3)$ values obtained experimentally for ${ }^{144-146} \mathrm{Ba}$ isotopes [35].

\section{CONCLUSIONS}

This paper reports on the first measurement of the $\beta$ decay of ${ }^{150} \mathrm{Cs} \rightarrow{ }^{150} \mathrm{Ba}$ and presents an extension of the partial level scheme for the ${ }^{148} \mathrm{Cs} \rightarrow{ }^{148} \mathrm{Ba}$ decay. They were measured at ISOLDE using the IDS setup which allowed also extraction of the lifetime for the $2_{1}^{+}$level in both daughter nuclei, using fast-timing techniques.

The level schemes for the two decays have been extracted from $\beta \gamma \gamma$ coincidences, and spins and parities have been proposed based on their decay pattern, $\beta$ feeding, and systematics. Low-spin positive- and negative-parity states have been effectively populated in both nuclei, confirming an interplay between GT and ff transitions.

A systematic evaluation of the low-lying levels in the $\mathrm{Ba}$ isotopic chain has been presented: the decrease in the excitation energy for the yrast band levels, with a parallel increase of the $B\left(E 2: 2^{+} \rightarrow 0^{+}\right)$transition probability, confirms an evolution towards increasing quadrupole deformation in these exotic members of the $\mathrm{Ba}$ isotopic chain. The low-spin pattern of the level scheme of ${ }^{150} \mathrm{Ba}$, showing positive- and negative-parity states connected by strong transitions, suggests the persistence of octupolar correlations, which have been established for the lighter $\mathrm{Ba}$ isotopes.
The experimental results have been compared to SCCM calculations with the Gogny D1S EDF including particle number, parity, and angular momentum restoration as well as quadrupole and octupole shape mixing. The calculations describe qualitatively the experimental excitation energies and transition probabilities in the whole isotopic chain ranging from $A=142$ to 150 . A sharper quadrupole shape transitionfrom almost spherical $\left({ }^{142} \mathrm{Ba}\right)$ to well prolate deformed nuclei ${ }^{144-150} \mathrm{Ba}$ - than that in the experimental data is obtained, and, in particular, theory also predicts a smaller quadrupole collectivity in ${ }^{142} \mathrm{Ba}$ and ${ }^{148-150} \mathrm{Ba}$. Octupole deformed ground states, with some softness along this degree of freedom, have been obtained in all of the isotopes studied here.

Future work to include triaxial and cranking intrinsic wave functions in the calculation is expected to return a better agreement with the experimental data.

\section{ACKNOWLEDGMENTS}

The authors are indebted to the late Prof. Henryk Mach whose experience helped in designing and performing the experiment. The IDS Collaboration acknowledges financial support from Istituto Nazionale di Fisica Nucleare, the Italian "Programmi di Ricerca Scientifica di Rilevante Interesse Nazionale" (PRIN), contract 2001024324 01302; the European Union seventh framework through ENSAR, contract 262010; the European Unions Horizon 2020 Framework research and innovation program under grant agreement 654002 (ENSAR2); the FATIMA-NuPNET network via the PRI-PIMNUP-2011-1338 project; the Romanian IFA grant CERN/ISOLDE and Romanian PN-II-RU-TE-2014-4-2003; the Spanish MINECO projects, reference numbers FPA201341467-P, FPA2015-64969-P, FPA2015-65929, and FIS201563770; Spanish grants FIS-2014-53434-P MINECO and Programa Ramon y Cajal 2012 No. 11420, MINECO grant IJCI-2014-19172, and the MINECO project FPA2014-52823C2-1-P; the German BMBF under contract 05P15PKCIA, contract 05P15PKFNA, and "Verbundprojekt 05P2015," the FWO-Vlaanderen (Belgium); and the IAP Belgian Science Policy (BriX network P7/12). V.Ch. and Z.P. acknowledge support by the Polish grant of Narodowe Centrum Nauki, no. 2015/18/M/ST2/00523.
[1] M. Dufour and A. P. Zuker, Phys. Rev. C 54, 1641 (1996).

[2] P. A. Butler and W. Nazarewicz, Rev. Mod. Phys. 68, 349 (1996).

[3] L. M. Robledo, M. Baldo, P. Schuck, and X. Vinas, Phys. Rev. C 81, 034315 (2010).

[4] H.-L. Wang, J. Yang, M.-L. Liu, and F.-R. Xu, Phys. Rev. C 92, 024303 (2015).

[5] J. L. Egido and L. M. Robledo, Nucl. Phys. A 524, 65 (1991).

[6] P. H. Heenen, J. Skalski, P. Bonche, and H. Flocard, Phys. Rev. C 50, 802 (1994).

[7] L. M. Robledo and G. F. Bertsch, Phys. Rev. C 84, 054302 (2011).

[8] P. Mason et al., Phys. Rev. C 72, 064315 (2005).

[9] W. Urban et al., Nucl. Phys. A 613, 107 (1997).
[10] J. Hamilton et al., Prog. Part. Nucl. Phys. 38, 273 (1997).

[11] J. C. Hill, F. K. Wohn, K. Leininger, J. A. Winger, M. E. Nieland, R. L. Gill, A. Piotrowski, R. F. Petry, and J. D. Goulden, Phys. Rev. C 34, 2312 (1986).

[12] B. Bucher et al., Phys. Rev. Lett 116, 112503 (2016).

[13] B. Bucher et al., Phys. Rev. Lett. 118, 152504 (2017).

[14] A. J. Mitchell et al., Phys. Rev. C 93, 014306 (2016).

[15] J. M. Yao, E. F. Zhou, and Z. P. Li, Phys. Rev. C 92, 041304(R) (2015).

[16] R. N. Bernard, L. M. Robledo, and T. R. Rodríguez, Phys. Rev. C 93, 061302(R) (2016).

[17] E. Kugler, Hyperfine Interact. 129, 23 (2000).

[18] J. Catherall et al., J. Phys. G: Nucl. Part. Phys. 44, 094002 (2017). 
[19] R. Lică et al., J. Phys. G: Nucl. Phys. 44, 054002 (2017).

[20] G. Rudstam et al., At. Data Nucl. Data Tables 53, 1 (1993).

[21] C. Chung, W. B. Walters, N. K. Aras, F. K. Wohn, D. S. Brenner, Y. Y. Chu, M. Shmid, R. L. Gill, R. E. Chrien, and L.-J. Yuan, Phys. Rev. C 29, 592 (1984).

[22] R. L. Gill et al., Phys. Rev. C 27, 1732 (1983).

[23] T. Kibédi et al., Nucl. Instrum. Methods Phys. Res., Sect. A 589, 202 (2008).

[24] W. Huang et al., Chin. Phys. C 41, 030002 (2017).

[25] M. Shmid et al., in Proceedings of the International Conference on Nuclei Far from Stability, Helsingor, Denmark, 1981 (CERN, Geneva, 1981), Vol. 2, p. 576.
[26] K. Okano et al., Z. Phys. A: Hadrons Nucl. 351, 243 (1995).

[27] H. Koura et al., Prog. Theor. Phys. 113, 305 (2005).

[28] T. Tachibana et al., Prog. Theor. Phys. 84, 641 (1990).

[29] H. Mach, W. Nazarewicz, D. Kusnezov, M. Moszyn‘ski, B. Fogelberg, M. Hellström, L. Spanier, R. L. Gill, R. F. Casten, and A. Wolf, Phys. Rev. C 41, R2469(R) (1990).

[30] M. Mariscotti, Phys. Rev. Lett. 24, 1242 (1970).

[31] R. Casten et al., Nucl. Phys. A 309, 477 (1978).

[32] L. Wilets et al., Phys. Rev. 102, 788 (1956).

[33] F. Berger, M. Girod, and D. Gogny, Nucl. Phys. A 428, 23 (1984).

[34] M. Borrajo et al., Phys. Lett. B 746, 341 (2015).

[35] G. Savard et al., Nucl. Instrum. Methods B 266, 4086 (2008). 